\title{
Corrigendum: A low-temperature method to produce highly reduced graphene oxide
}

\author{
Hongbin Feng, Rui Cheng, Xin Zhao, Xiangfeng Duan \& Jinghong Li
}

Nature Communications 4:1539 doi: 10.1038/ncomms2555 (2013); Published 26 Feb 2013; Updated 8 Jul 2013

This Article contains errors in Fig. 3. The Raman spectra that were included in Fig. 3d while the manuscript was under consideration by the journal were inadvertently replaced with Fourier transform infrared (FTIR) spectra during the final revision. The correct version of Fig. 3 appears below.

a

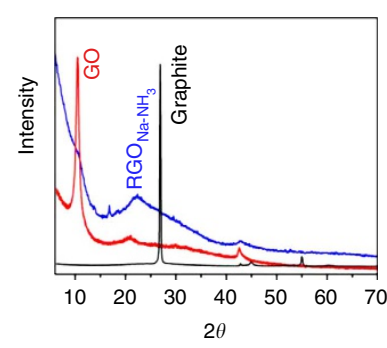

C

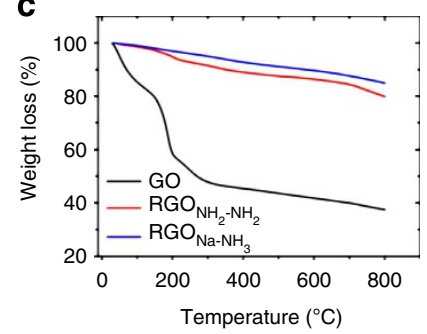

b
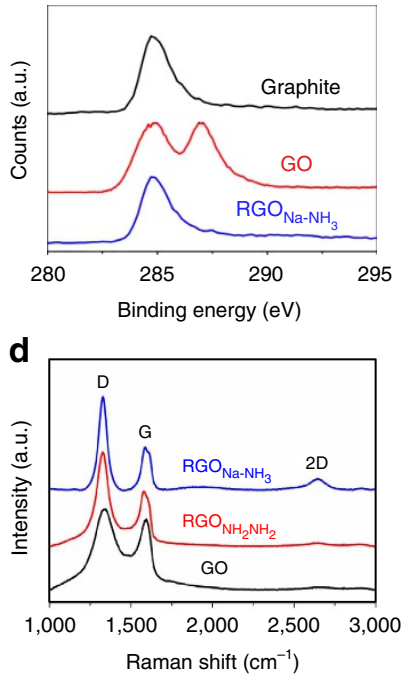